\title{
A Large-capacity Information Hiding Method for HEVC Video
}

\author{
Wang Jiaji, Wang Rangding, Li Wei, Xu Dawen, Huang Meiling \\ CKC Software Lab \\ Ningbo University \\ Ningbo, China \\ Corresponding Author e-mail: wangrangding @nbu.edu.cn
}

\begin{abstract}
A large-capacity information hiding algorithm for HEVC is proposed. We modify the intra prediction modes in $4 \times 4$ luminance blocks to embed secret information. According to the probability distribution of the statistical optimal prediction mode and suboptimal prediction mode, we are establishing a mapping relationship between prediction modes and secret information. Modifying the intra prediction modes to embed secret information is based on the mapping. The extraction of information merely requires decoding the prediction mode from the bit stream. Experimental results show that the proposed hiding algorithm can embed information with large capacity and effectively guarantee the subjective and objective quality of video.
\end{abstract}

Keywords-information hiding; HEVC; intra prediction; mapping

\section{INTRODUCTION}

With the development of the digital multimedia and network technology, the application and distribution of digital information is indispensable to our daily life. Simultaneously, the digital information is counterfeited, tampered, and attacked, which are increasingly serious phenomenon [1]. Hence, information security problem is increasingly highlighted. Information hiding technology plays an important role in curbing the illegal use of digital multimedia, protecting information from the interference and damage.

HEVC standard is a next-generation video coding standard which is developed by ITU-T Video Coding Experts Group and Moving Picture Experts Group [2]. It is aimed to significantly improve encoding efficiency on the basis of H.264/AVC, especially for dealing with highdefinition video. The efficient compression performance and processing capacity of HEVC standard have an important role in high-definition video applications. The information hiding algorithm for HEVC has a theoretical value and practical significance. At present, the information hiding algorithm based on H.264/AVC has become mature, such as those based on the modified DCT coefficients, motion vector and prediction [4-8]. However, based on HEVC is still in its infancy. Chang et al. [3] presented a hiding algorithm for HEVC based on DCT/DST coefficients. It has the advantage of reducing the intra-frame error propagation-free and improving the visual effect of video.

Among the information hiding algorithms for H.264/AVC based on the intra prediction mode, $\mathrm{Hu}$ et al. [4] modified the intra prediction mode based on the mapping between the secret information and the prediction mode. The mapping relationship is established on the statistical results of multiple video test sequences. Wang et al. [5] embedded secret information in I-frame, P-frame and B-frame through adjustment of the encoding mode of given macroblocks. Xu et al. [6] proposed a hiding information algorithm based on modulating the intra prediction of $4 \times 4$ luminance blocks. If the best mode does not match the information bit, the best prediction mode will be replaced with the substitute mode which is the one with the least Lagrangian cost among those having different parity with the best mode. Yang et al. [7] established a mapping between the information and the intra prediction mode with matrix coding. Two secret information bits are inserted in three intra-frame $4 \times 4$ luminance blocks only modify a prediction mode. Yin et al. [8] proposed an algorithm based on Yang's work [7], and utilized an embedding/ extracting matrix, three watermark bits are embedded while only one intra- $4 \times 4$ block's mode is changed.

In order to improve the embedding capacity under the premise of ensuring the video subjective and objective quality, a large-capacity information hiding algorithm for HEVC is proposed. We are establishing a mapping relationship between prediction modes and hiding information. Modifying the intra prediction modes to embed secret information is based on the mapping. The extraction of information merely requires decoding the prediction mode from the bit stream. The information hiding algorithm is satisfied with the needs of real-time performance.

\section{INTRA-PREDICTION IN HEVC}

Similar to the H.264/AVC, HEVC adopts the conventional hybrid video coding framework [9]. However, compared to the H.264/AVC, HEVC gives up the concept of macroblock but introduces three basic uints: coding unit(CU), prediction unit(PU)and transform unit(TU).The separation of three basic units can make the procession of prediction, transformation and coding more flexible. The effect after compression can be more in line with the characteristics of video image itself.

In H.264/AVC standard, intra prediction coding exploits the spatial correlation of pixels. The pixels of the current block are predicted by adjacent pixels in the neighboring blocks that are coded and reconstructed earlier [10]. The selection process of best intra prediction mode uses the Lagrangian rate-distortion optimization model to choose the minimum rate-distortion cost from all the intra prediction modes. In principle, intra prediction of HEVC adopts the same technology in H.264/AVC, but increases the number of 
the prediction mode. In Fig. 1(a), there are nine intra prediction modes of $4 \times 4$ luminance block in H.264/AVC. However, HEVC refines more prediction modes and provides as many as 35 intra prediction modes, as is showed in Fig. 1(b). The increased model number makes intra prediction more accurate and reduces the spatial redundancy [11].

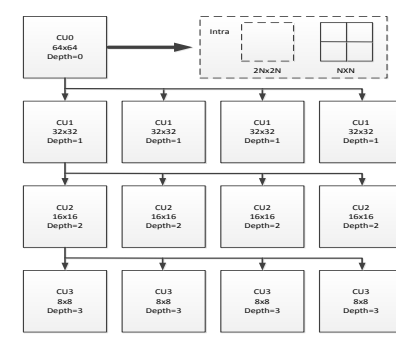

(a)

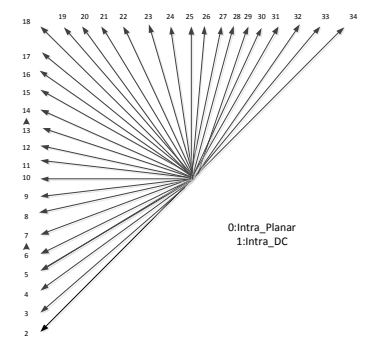

(b)
Figure 1. Prediction modes for H.264/AVC and HEVC.

To select the reasonable and effective optimal mode from the 35 prediction modes, HEVC chooses the optimal prediction mode by using the Lagrangian optimization mode. The computation formula is computed by:

$J\left(s, c, I M O D E \mid Q P, \lambda_{M O D E}\right)=D(s, c, I M O D E \mid Q P)+\lambda_{M O D E} \cdot R(s, c, I M O D E \mid Q P)$,

where $Q P$ is the quantization parameter and $\lambda_{M O D E}$ is the Lagrange multiplier. $S$ and $c$ represent the original block and reconstruction block respectively. $R$ is bit-rate and $D$ is the distortion degree.

\section{INFORMATION HIDING ALGORITHM}

In this paper, the intra prediction modes in $4 \times 4$ luminance blocks are modified to embed secret information. According to the mapping relationship between information bits and prediction modes, the corresponding prediction mode is modified to complete the information embedding.

\section{A. The Principle of Information Hiding}

Modifying the prediction mode will affect the quality of the video. If the difference between the optimal prediction mode and the substitutive prediction mode is obvious, it will have a great influence on the subjective and objective quality of video. When the prediction mode is modified to embed information, we hope to change the optimal prediction mode to the sub-optimal prediction mode which is close to the original video effect, if possible. Considering the correlation between the prediction modes, when the optimal prediction mode $M_{i}(i=0,1, \cdots, 34)$ is determined, analyze the distribution probability of the sub-optimal prediction mode. This paper shows the statistical probability distribution of the sub-optimal prediction modes of test sequence with different resolution. As shown in Fig. 2, a part of the statistical results are presented. Fig.4 (a), (b), (c), (d) present the probability distribution of the sub-optimal prediction mode when the optimal mode is $M_{i}(i=8,9,10,11)$.

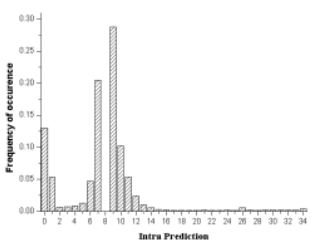

(a) Optimal prediction mode 8

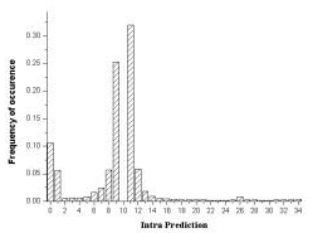

(c) Optimal prediction mode 10

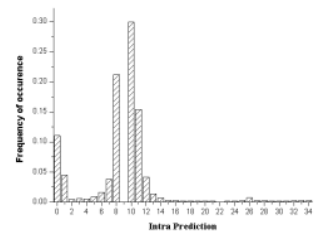

(b) Optimal prediction mode 9

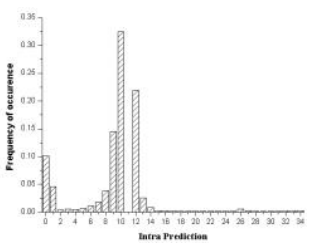

(d) Optimal prediction mode 11
Figure 2. When the optimal prediction mode is determined, the probability of the sub-optimal prediction mode distribution

The probability of the prediction mode which is nearby the optimal prediction mode becomes the sub-optimal prediction mode is very high. In other words, similar angle prediction modes have strong spatial correlation. In this paper, we modify the intra prediction mode based on the mapping between the secret information and the prediction with the sub-optimal prediction distribution. To improve the embedding capacity, we propose a novel method that modifying one prediction mode can embed two bits secret information. For four prediction modes which have similar prediction effect, the 35 prediction modes are divided into groups which have four alternative modes.

According to Fig. 2, considering the spatial correlation and the degree of overlap among the prediction modes, for the optimal prediction mode $M_{i}$, we choose the high probability of the top five of prediction modes $\left(N_{1}, N_{2}, N_{3}, N_{4}, N_{5}\right)$ and $M_{i}$ to form a set $G_{i}(i=0,1, \cdots, 34)$ which contains six elements. As shown in Fig. 2(a), the optimal prediction 8 and the high probability of the first five prediction modes 9,7,1,10,0 form the set $G_{8}=\{8,9,7,1,10,0\}$. We randomly select four sets from the 35 sets and get intersection elements. When the number of the intersection elements is more than 3, the optimal prediction mode of the four sets form a prediction mode group. The computation formula is computed as:

$C\left(G_{i}, G_{j}, G_{m}, G_{n} \mid m, n, i, j\right)=\left(G_{i} \cap G_{j} \cap G_{m} \cap G_{n} \mid m \neq n \neq i \neq j, 0 \leq m, n, j, i \leq 34\right)$,

where $G_{i}, G_{j}, G_{m}, G_{n}$ are four different sets, and $C$ is the results of intersection calculation for four sets.

In conclusion, we can get 11 prediction mode groups as shown in TABLE I and establish the mapping relationship between the secret information and prediction mode. For each group, group $j(1 \leq j \leq 11)$ contains four modes which 
named $N_{1}, N_{2}, N_{3}, N_{4}$. As shown in TABLE II, modify the corresponding prediction mode according to the value of the secret information. For example, the prediction mode of the $4 \times 4$ luminance block is $N_{1}$, two information bits are 0 and 1 . On the basis of the mapping table, we change the prediction mode into $\mathrm{N}_{2}$ to embed information.

TABLE I. PREDICTION MODE GROUP

\begin{tabular}{|c|c|}
\hline Group & Intra Prediction \\
\hline Group 1 & $2,3,4,0$ \\
\hline Group 2 & $5,6,7,0$ \\
\hline Group 3 & $8,9,10,11$ \\
\hline Group 4 & $12,13,0,1$ \\
\hline Group 5 & $14,15,16,0$ \\
\hline Group 6 & $17,18,19,0$ \\
\hline Group 7 & $20,21,22,0$ \\
\hline Group 8 & $23,24,25,0$ \\
\hline Group 9 & $26,27,28,0$ \\
\hline Group 10 & $29,30,0,1$ \\
\hline Group 11 & $31,32,33,34$ \\
\hline
\end{tabular}

TABLE II. PREDICTION MODE GROUP

\begin{tabular}{|c|c|}
\hline Prediction Mode & Secret Information \\
\hline $\mathrm{N}_{1}$ & 00 \\
\hline $\mathrm{N}_{2}$ & 01 \\
\hline $\mathrm{N}_{3}$ & 10 \\
\hline $\mathrm{N}_{4}$ & 11 \\
\hline
\end{tabular}

For all of 11 groups in TABLE I, we can establish the group mapping relationship between the information and the prediction modes to embed secret information in order to get the biggest hiding capacity. Considering reducing the video quality decline caused by embedding secret information, we only choose a few prediction mode groups to embed information. In order to keep the hiding capacity, we select test sequence with different resolution to do the statistical probability distribution of the intra prediction mode. As shown in Fig. 3, we choose a high probability of prediction mode for modulation and choose Group 3, 4, 7, 9, 10 to embed information.

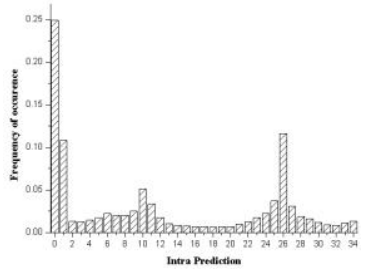

Figure 3. The probability distribution of the optimal prediction mode

\section{B. Data Embedding Procedure}

In the proposed method, we embed the secret information according to the mapping between the prediction and the information. The hiding algorithm is described as following:

Step1. For the $4 \times 4$ luminance block, extract the optimal prediction mode and find the corresponding group through the TABLE I.

Step2. According to the mapping between the secret information and the prediction mode, we replace the optimal prediction mode with the corresponding prediction of the secret information, and re-encode the $4 \times 4$ luminance block with the substitutive prediction mode.

Step3. Compare the $4 \times 4$ blocks with other encoding modes with the Lagrangian cost. If the cost of the $4 \times 4$ blocks is less than the other encoding modes, save the embedded information. Otherwise, embed the current bits next time without saving the embedded bits.

Step4. Repeat the above steps until embedding all the secret information.

\section{Data Detection Procedure}

Secret data detection procedure is simple, only need to decode part of I frame. The steps are described as following: Step 1. Determine whether the block coding mode of current block is $4 \times 4$ type. If the current block coding mode is other coding mode, go to step3 without extracting the secret information. If the block coding mode is $4 \times 4$ type, decode the prediction of current block.

Step 2. Decode the prediction of $4 \times 4$ luminance block. We extract the information according to the mapping between the prediction and the secret information.

Step 3. Repeat the Step1 and Step 2 for the $4 \times 4$ blocks until extracting all the information.

\section{EXPERIMENTAL RESULTS AND DISCUSSIONS}

This algorithm has been conducted on the HEVC HM12.0 reference software, experimented with different kinds of test sequence. The main reference software coding configuration parameters are shown in TABLE III, and the remaining parameters are set to the default configuration.

TABLE III. CONFIGURATION PARAMETERS OF THE HM

\begin{tabular}{|c|c|}
\hline Parameters & Configuration \\
\hline Frames To Be Encoded & 96 \\
\hline Frame Rate & $30 \mathrm{fps}$ \\
\hline Intra Period & 16 \\
\hline GOPSize & 8 \\
\hline RDOQTS & 1 \\
\hline
\end{tabular}

Experiments evaluate algorithm from the objective video coding quality variation $(P S N R)$, bit rate variation $(B R I)$ and data hiding capacity $(H B Q)$. BRI and $P S N R$ are defined as follows:

$B R I=\frac{R^{\prime}-R}{R} \times 100(\%)$

$P S N R=P S N R_{Y}^{\prime}-P S N R_{Y}$

where $R$ and $R$ is the bit rate before and after embedded secret information, $P S N R_{Y}^{\prime}$ and $P S N R_{Y}$ is the video coding quality before and after embedded secret information. 
TABLE IV lists the experimental results of the comprehensive performance. Through analyzing the data results, we can draw the following conclusions:

For different video test sequence, the number of $4 \times 4$ luminance block leads to the difference of embedding information capacity.

The PSNR and bit rate of the original and embedded video changes little, indicating that the algorithm induces limited quality degradation. It satisfies the visual concealment of the information hiding.

TABLE IV. EXPERIMENTAL RESULTS OF THE COMPREHENSIVE PERFORMANCE

\begin{tabular}{|l|l|l|l|}
\hline Sequence & BRI(\%) & PSNR & HBQ(bits) \\
\hline BasketballDrill & 2.94 & -0.02 & 29778 \\
\hline Vidyo1 & 2.12 & -0.01 & 22312 \\
\hline Vidyo3 & 2.44 & -0.03 & 28240 \\
\hline Vidyo4 & 1.78 & -0.01 & 19820 \\
\hline
\end{tabular}

To evaluate the algorithm performance, Xu et al. [6]'s algorithm is conducted on the HEVC HM-12.0 reference software. Fig. 4 5 presents the comparison with the algorithms in the proposed method and [6]. Fig. 4(a) shows the comparison of the hiding capacity. Fig. 4(b) shows the comparison of the bit rate. Fig. 5shows the PSNR difference between [6].

As can be seen from the diagram, the bit rate increases a little and the PSNR loss is just about $0.01 \mathrm{~dB}$. The hiding information capacity has greatly increased, nearly double the hiding capacity.

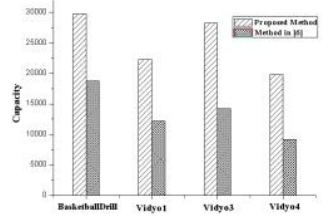

(a)

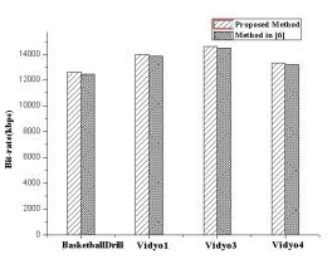

(b)
Figure 4. Comparison of the hiding capacity and bit-rate

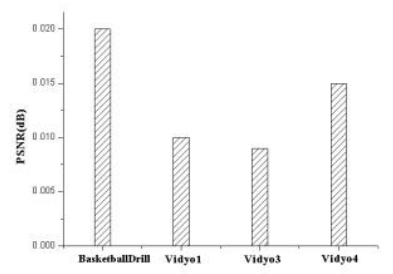

Figure 5. The PSNR difference between method [6]

\section{CONCLUSIONS}

In this paper, an information hiding algorithm of large capacity for HEVC is proposed. The intra prediction modes in $4 \times 4$ luminance blocks are modified to embed secret information. The algorithm embeds secret information by modifying the intra prediction modes based on the mapping relationship, which is established between prediction modes and secret information, according to the probability distribution of the statistical optimal prediction mode and suboptimal prediction mode. The extraction of information merely requires decoding the prediction mode from the bit stream. Experiments show that the algorithm extraction process is simple and rapid, while improve the embedding capacity on the promise of ensuring the subjective and objective quality of the video. There is little impact on the video after embedding information.

\section{ACKNOWLEDGMENT}

This work is sponsored by K.C. Wong Magna Fund in Ningbo University, National Natural Science Foundation of China (NSFC: 61170137, 61300055, 61301247), Doctoral Fund of Ministry of Education of China(20103305110002), Zhejiang natural science foundation of China (ZJNSF: LY13F020013), Ningbo natural science foundation of China (2013A610057, 2013A610059), Scientific Research Fund of Zhejiang Provincial Education Department (Y201119434), Open Fund of Zhejiang Provincial Top key Discipline of Information and Communication Engineering(XKXL1313, XKXL1310).

\section{REFERENCES}

[1] COX I J, MILLER M L, BLOOM J A. Digital Watermarking. Elsevier Science, USA, 2002.

[2] Han G J, Ohm J R, Han W J, "Overview of the high efficiency video coding (HEVC) standard," 2012, vol22, pp.1649-1668.

[3] Chang P C, Chung K L, Chen J J, "A DCT/DST-based error propagation-free data hiding algorithm for HEVC intra-coded frames," unpublished..

[4] HU Yang, ZHANG Chun-tian, SU Yu-ting, "Information hiding for H.264/AVC," Acta Electronica Sinica, 2008, vol36, pp. 690-694.

[5] WANG Rang-ding, ZHU Hong-liu, XU Da-wen, "Information hiding algorithm for H.264/AVC based on encoding mode," Opto-Electronic Engineering, 2010, vol37, pp: 144-150.

[6] Xu D W, Wang R D, Wang J C, "Prediction mode modulated datahiding algorithm for H.264/AVC," Journal of Real-Time Image Processing, 2012, vol7, pp. 205-214.

[7] Yang G B, Li J J, He Y L, Kang Z W, "An information hiding algorithm based on intra-prediction modes and matrix coding for H.264/AVC video stream," AEU-International Journal of Electronics and Communications, 2011, vol65, pp. 331-337.

[8] YIN Qiu-lai, Wang Hong-xia, Zhao Yan, "An information hiding algorithm based on intra-prediction modes for H.264 video stream," Journal of Optoelectronics'Laser, 2012, vol23, pp.2194-2199.

[9] Ohm J, Sullivan G J, Schwarz H, "Comparison of the coding efficiency of video coding standards - including high efficiency video coding (HEVC)," Circuits and Systems for Video Technology, IEEE Transactions on, 2012, vol22, pp. 1669-1684.

[10] Lainema J, Bossen F, Han W J, "Intra coding of the HEVC standard," Circuits and Systems for Video Technology, IEEE Transactions on, 2012, vol22, pp.1792-1801.

[11] Kim I K, Min J, Lee T, "Block partitioning structure in the HEVC standard," Circuits and Systems for Video Technology, IEEE Transactions on, 2012, vol22, pp.1697-1706. 